\title{
Association between Exosomal miRNAs and Coronary Artery Disease by Next-Generation Sequencing
}

\author{
Sheng-Nan Chang ${ }^{1}{ }^{\oplus}$, Jien-Jiun Chen ${ }^{1}$, Jo-Hsuan $W u^{2}{ }^{\circledR}$, Yao-Te Chung ${ }^{1}$, Jin-Wun Chen ${ }^{1}$, Chu-Hsuan Chiu ${ }^{3,4}$, \\ Chia-Ju Liu ${ }^{5}{ }^{\circ}$, Meng-Tsun Liu ${ }^{1}$, Yi-Cheng Chang ${ }^{3,4}{ }^{\oplus}$, Chin Li ${ }^{6}{ }^{(}$, Jou-Wei Lin ${ }^{1, *}{ }^{\mathbb{C}}$, Juey-Jen Hwang ${ }^{1,7}$ \\ and Wen-Pin Lien ${ }^{7}$
}

\section{check for} updates

Citation: Chang, S.-N.; Chen, J.-J.; Wu, J.-H.; Chung, Y.-T.; Chen, J.-W.; Chiu, C.-H.; Liu, C.-J.; Liu, M.-T.; Chang, Y.-C.; Li, C.; et al. Association between Exosomal miRNAs and Coronary Artery Disease by NextGeneration Sequencing. Cells 2022, 11, 98. https://doi.org/10.3390/ cells11010098

Academic Editor:

Kay-Dietrich Wagner

Received: 21 November 2021

Accepted: 27 December 2021

Published: 29 December 2021

Publisher's Note: MDPI stays neutral with regard to jurisdictional claims in published maps and institutional affiliations.

Copyright: (C) 2021 by the authors. Licensee MDPI, Basel, Switzerland. This article is an open access article distributed under the terms and conditions of the Creative Commons Attribution (CC BY) license (https:// creativecommons.org/licenses/by/ $4.0 /)$.
1 Division of Cardiology, Department of Internal Medicine, National Taiwan University Hospital Yunlin Branch, Douliu City 640203, Taiwan; p95421008@ntu.edu.tw (S.-N.C.); jienjiunc@ntu.edu.tw (J.-J.C.); y00703@ms1.ylh.gov.tw (Y.-T.C.); a0988890276@gmail.com (J.-W.C.); drliumt168@hotmail.com (M.-T.L.); jueyhwang@ntu.edu.tw (J.-J.H.)

2 Shiley Eye Institute and Viterbi Family Department of Ophthalmology, University of California, San Diego, CA 92093, USA; uuuuuu1swu@gmail.com

3 Graduate Institute of Medical Genomics and Proteomics, National Taiwan University, Taipei 100, Taiwan; victor26aaaa@ibms.sinica.edu.tw (C.-H.C.); b83401040@gmail.com (Y.-C.C.)

4 Institute of Biomedical Sciences, Academia Sinica, Taipei 115, Taiwan

5 Department of Nuclear Medicine, National Taiwan University Hospital, Taipei 100, Taiwan; chiaju.liu@gmail.com

6 Department of Biomedical Sciences, National Chung-Cheng University, Chia-Yi 62102, Taiwan; biocl@ccu.edu.tw

7 Department of Internal Medicine, National Taiwan University Hospital, Taipei 100, Taiwan; jeremysnc1000@gmail.com

* Correspondence: jouweilin@yahoo.com; Tel.: +886-922-861-953; Fax: +886-5-537-3257

\begin{abstract}
Background: Among various bio-informative molecules transferred by exosomes between cells, micro RNAs (miRNAs), which remain remarkably stable even after freeze-and-thaw cycles, are excellent candidates for potential biomarkers for coronary artery disease (CAD). Methods: Blood samples were collected from the coronary arteries of 214 patients diagnosed with three-vessel CAD and 140 without CAD. After precipitation extraction, the amounts of exosomes were found to decrease with increased age and three-vessel CAD. Next-generation sequencing was performed to further explore the possible relationship between exosomal miRNAs and CAD. Results: Eight exosomal miRNAs showed altered expression associated with CAD. The up-regulated miRNAs in CAD were miRNA-382-3p, miRNA-432-5p, miRNA-200a-3p, and miRNA-3613-3p. The downregulated miRNAs were miRNA-125a-5p, miRNA-185-5p, miRNA-151a-3p, and miRNA-328-3p. Conclusion: We successfully demonstrated particular exosomal miRNAs that may serve as future biomarkers for CAD.
\end{abstract}

Keywords: coronary artery disease; exosomes; micro RNAs; next-generation sequencing

\section{Introduction}

Exosomes are actively secreted membrane vesicles that are recognized as the major mediators of intercellular communication [1]. They are 40-100 $\mathrm{nm}$ in size and can transfer messenger RNAs (mRNAs), micro RNAs (miRNAs), non-coding RNAs, transcription factors, and proteins between cells [2]. In eukaryotic animals, these extracellular organelles are released into the microenvironment in response to microenvironmental stimuli for cell-to-cell communication [3].

As previously reported, chronic inflammation is involved in the pathogenetic process of atherosclerosis. Some inflammation activities, including proteolysis, calcification, and neovascularization, can lead to the development of atherosclerotic plaques on the arteries, which is diagnosed clinically as coronary artery disease (CAD) [4]. In response to 
these inflammatory stimuli, the exosomes might be secreted and cause changes within the cardiomyocytes [5].

Among various genetic molecules within the exosomes, miRNAs are one of the most attended and studied elements. miRNAs can remain stable even after freeze-and-thaw cycles, making them excellent candidates for potential biomarkers for CAD [6]. However, little is known about the role of exosomal miRNAs in CAD. With the hypothesis of a possible change in the amounts and contents of exosomes associated with CAD, the current study investigated the altered expression of miRNAs in the pathophysiology of CAD for further clinical application.

\section{Materials and Methods}

\subsection{Study Population}

In this study, 625 patients were enrolled from the National Taiwan University Hospital Yun-Lin Branch, Taiwan, between January 2018 and September 2021. The inclusion criteria were patients hospitalized for elective coronary artery angiography under the impression of stable CAD rather than admitted due to acute coronary syndrome (ACS) [7]. The exclusion criteria were ACS, cardiac arrhythmias, chronic obstructive pulmonary disease, thyroid disease, rheumatic arthritis, malignancy, hepatitis B infection, hepatitis $C$ infection, hemoglobin $\leq 10 \mathrm{~g} / \mathrm{dL}$, albumin $\leq 3.2 \mathrm{~g} / \mathrm{dL}$, aspartate transaminase $\geq 100$ units $/ \mathrm{L}$, alanine aminotransferase $\geq 100$ units $/ \mathrm{L}$, or serum creatinine $\geq 2 \mathrm{mg} / \mathrm{dL}$.

Three-vessel CAD was defined by coronary angiography as $\geq 50 \%$ stenosis of the left anterior descendent branch, left circumflex branch, right coronary artery, and/or major branches of each artery in combination with/without the left main coronary artery [8]. The diagnosis of three-vessel CAD was confirmed by coronary angiography, and all the results were interpreted by two cardiologists. The blood samples were collected from the coronary artery during coronary angiography before percutaneous coronary intervention if necessary, and the precipitation method was used for exosome isolation in both participants with/without three-vessel CAD. The amounts of exosomes were calculated by flow cytometry and subsequently verified by Western blot. This study was approved by the Ethics Committee and Institutional Review Board on Human Research of the Medical Research Department of National Taiwan University Hospital, Taipei, Taiwan (201811060RINC/202001100RINB) and adhered to the Declaration of Helsinki. All subjects provided written informed consent before participating in the study. The study workflow is summarized in Supplementary Figure S1.

\subsection{Blood Samples Collection}

Initially, the blood samples were collected in EDTA tubes $(10 \mathrm{~mL})$. The blood samples were later centrifugated $\left(3000 \times g, 4{ }^{\circ} \mathrm{C}\right.$, for $\left.10 \mathrm{~min}\right)$ to collect plasma. The plasma was collected in AXYGEN microtubes (MCT-175-C) and stored at $-70{ }^{\circ} \mathrm{C}$ for further exosome extraction.

\subsection{Method for Precipitation Extraction}

The plasma $(2.5 \mathrm{~mL})$ was centrifuged $\left(3000 \times g, 4^{\circ} \mathrm{C}\right.$, for $\left.15 \mathrm{~min}\right)$ to remove cells and cell debris. The supernatant was transferred and filtered with a $0.22 \mu \mathrm{m}$ membrane (Millipore, Burlington, MA, USA) to a sterile vessel $(900 \mu \mathrm{L})$. After that, an appropriate volume of exosome precipitation solution $(300 \mu \mathrm{L})$ (ExoQuick, System Biosciences, Mountain View, CA, USA) was added to the bio-fluid. The sample tubes remained upright and refrigerated (at $4{ }^{\circ} \mathrm{C}, 30 \mathrm{~min}$ ). Of note, the tubes should not be rotated or mixed during the incubation period and should remain upright during these steps. The vessels were then centrifuged $\left(1500 \times g, 4{ }^{\circ} \mathrm{C}\right.$, for $\left.30 \mathrm{~min}\right)$. After centrifugation, the exosomes may appear as a beige or white pellet at the bottom of the vessels. The resulting exosome pellet was resuspended in $600 \mu \mathrm{L}$ sterile $1 \times$ PBS with a $0.22 \mu \mathrm{m}$ membrane filter (Millipore, Merck KGaA, Darmstadt, Germany). The exosome pellets were resuspended in $600 \mu \mathrm{L}$ sterile $1 \times$ PBS for further analysis [9]. 


\subsection{Characterization of the Samples of Exosomes}

To characterize the samples of exosomes, flow cytometry and Western blot assays were used, and equal protein concentration loading and exosome markers were applied. The particle sizes were assessed with flow cytometry. The standard nanoparticles of $0.1 \mu \mathrm{m}$, $0.2 \mu \mathrm{m}, 0.5 \mu \mathrm{m}$, and $0.8 \mu \mathrm{m}$ were used to establish the particle size analysis. The Western blot assays were used to detect the exosome markers. Specific markers for exosomes, including CD9, CD63, CD81, and Hsp70, were utilized in this study, and as expected, the standard control markers were found in the exosomes [10].

\subsubsection{Flow Cytometric Analysis}

The exosomes were filtered with a $0.22 \mu \mathrm{m}$ membrane (Millipore, Merck KGaA, Darmstadt, Germany) and precipitated. The purified exosomes were suspended in $1 \times$ phosphatebuffered saline (PBS). The solution was diluted to $10^{4}$-fold in the $1 \times$ PBS assay for the flow cytometry (CytoFLEX BB10068, Beckman coulter, Brea, CA, USA). The flow cytometry was set to: (1) sample flow rate: $10 \mu \mathrm{L} / \mathrm{mL}$; (2) events to record: 50,000 events at $0.1 \mu \mathrm{m}$; (3) time to record: $600 \mathrm{~s}$; (4) volume to record: $10 \mu \mathrm{L}$. Because PBS was used for exosome suspension, we used PBS as the reference signal in the flow cytometric analysis [11].

\subsubsection{Exosomes Assays by Western Blot}

The exosomes were lysed in ice-cold $5 \times$ lysis reagent (Promega, Madison, WI, USA). The protein concentration was quantified by Bradford reagent (Sigma, Merck KGaA, Darmstadt, Germany). The $10 \%$ sodium dodecyl sulfate polyacrylamide gel electrophoresis (SDS-PAGE) was prepared for protein electrophoresis. Total proteins were mixed with $5 \times$ sampling buffer and boiled at $100{ }^{\circ} \mathrm{C}$ for $10 \mathrm{~min}$. The mixture and protein markers were loaded in wells for SDS-PAGE at $50 \mathrm{~V}$ and $110 \mathrm{~V}$ for protein electrophoresis. After protein electrophoresis, separated proteins were transferred to a polyvinylidene difluoride (PVDF) membrane Millipore, Merck KGaA, Darmstadt, Germany) at $95 \mathrm{~V}$ for 2 h, and then, 5\% skim milk (Sigma, Merck KGaA, Darmstadt, Germany). blocking solution for $1 \mathrm{~h}$ at $4{ }^{\circ} \mathrm{C}$ with shaking was used for the PVDF membrane. After blocking, the exosome markers were prepared in $1 \times$ PBS with $0.05 \%$ PBS, $0.1 \%$ Tween (PBST) (Sigma, Merck $\mathrm{KGaA}$, Darmstadt, Germany), and $5 \%$ skim milk overnight at $4{ }^{\circ} \mathrm{C}$ under shaking. We used anti-CD9 antibody (1:1000) (SBI, CA), anti-CD63 antibody (1:20,000) (SBI, CA), anti-CD81 antibody (1:1000) (Abcam, Cambridge, UK), and anti-Hsp70 antibody (1:1000) (SBI, CA) for exosome identification and confirmation. The Bradford method was used for evaluating the quantities of samples for gel electrophoresis. The samples were normalized to the same protein volumes. The catalog numbers and concentrations of the loading proteins were CD9 (5 $\mu \mathrm{g}$, EXOAB-CD9A-1), CD63 (EXOAB-CD63A-1, $5 \mu \mathrm{g})$, CD81 (10 $\mu \mathrm{g}, \mathrm{ab109201})$, and Hsp70 (5 $\mu \mathrm{g}$, EXOAB-Hsp70A-1) [12].

After primary antibody incubation, membranes with transferred proteins were washed with PBST four times. The secondary antibody (anti-rabbit, 1:10,000) (Millipore, USA) was prepared in PBST with 5\% skim milk for $2 \mathrm{~h}$ at room temperature under shaking. After the secondary antibody was discarded, the membranes were washed with PBST four times. The enhanced chemiluminescence detection kit (ECL; PerkinElmer, Waltham, MA, USA) was used for identifying the protein bands.

\subsection{Illumina Next-Generation Sequencing}

RNA from purified exosomes was purified using TRIzol reagent. Glycogen was used to facilitate the recovery of small RNA. The specimens were converted to the sequencing library using the Small RNA-Seq Library prep Kit (Lexogen, Vienna, Austria). The manufacturer' s protocol was closely followed to generate the library. Briefly, purified RNA was ligated to the 10 -fold diluted $3^{\prime}$-adapter in the first ligation reaction followed by a column-based purification process to remove excess adaptor. The $5^{\prime}$-adaptor was diluted 10 -fold and denatured by heating before ligating to purified small RNA with the $3^{\prime}$-adaptor in the second ligation reaction. Adaptor-ligated RNA was converted to cDNA by reverse 
transcription and amplified by PCR. The library was further analyzed by non-denaturing polyacrylamide gel and enriched by eluting the desired size DNA library from the gel. The eluted library was subjected to a limited PCR cycle to obtain a sufficient concentration for sequencing in an Illumina NextSeq 550 sequencer for 50 cycles. The sequencing reads were imported to CLC genomic Workbench 21.0.3. Micro RNA was identified by mapping against the miRBase database 22.1. The result was then exported for statistical analysis [13].

\subsection{Statistical Analyses}

Statistical analyses were performed by a researcher who was blinded to the subjects' conditions. Continuous variables are presented as the mean \pm standard deviation (SD) and were compared with a two-tailed Student $t$-test. The categorical variables are reported as frequencies or percentages. The non-normal variables are presented as the median with the interquartile range and were compared with the Mann-Whitney U-test. Multiple linear regression analyses were used to measure the association between the amounts of exosomes and possible co-variables such as age, sex, coronary artery disease, hypertension, and diabetes mellitus. The miRNA expression levels were compared using the Student $t$-test. A two-tailed $p$-value $<0.05$ was considered statistically significant. The statistical analyses were performed by the SPSS Version 25.0 statistical software (SPSS, Chicago, IL, USA).

\section{Results}

Among the 625 patients' post-coronary intervention, 214 patients were diagnosed with three-vessel CAD and categorized into the CAD group. Another 140 patients who were confirmed not to have CAD after coronary angiography were categorized into the patient group. The clinical and laboratory characteristics of these two groups are presented in Table 1. Generally, the mean ages of both groups were over sixty years old, and the patients with three-vessel CAD were older than the patients without CAD (CAD vs. patient: $66.83 \pm 10.96$ vs. $60.82 \pm 11.67 ; p<0.001$ ) (Table 1). Both groups were predominantly male with more than 50\% male participants (CAD: 77\%; patient: 69\%) (Table 1). In terms of weight, the BMI values were similar between both groups (CAD vs. patient: $26.04 \pm 3.93$ vs. $26.75 \pm 4.00 ; p=0.1$ ) (Table 1 ). For the history of systemic diseases, there was a greater percentage of hypertension (HTN) (CAD vs. patient: $70 \%$ vs. $54 \% ; p=0.03$ ) and diabetes mellitus (DM) (CAD vs. patient: $46 \%$ vs. $26 \%$; $p=0.02$ ) diagnosis in the CAD group (Table 1). However, the systolic blood pressure (SBP) (CAD vs. patient: $135.43 \pm 26.68$ vs. $138.98 \pm 24.64 ; p=0.21)$ and the hemoglobin A1c (HbA1c) level were not significantly different (CAD vs. patient: $6.47 \pm 1.38$ vs. $6.38 \pm 1.75 ; p=0.84$ ) (Table 1 ). The diastolic blood pressure (DBP) was lower in the CAD group (CAD vs. patient: $71.68 \pm 14.56 \mathrm{vs}$. $75.48 \pm 13.01 ; p=0.01$ ) (Table 1). The mean creatine level of three-vessel CAD patients was higher (CAD vs. patient: $1.47 \pm 1.86$ vs. $0.99 \pm 0.57 ; p<0.001$ ), but the appearance of proteinuria did not differ between the two groups (CAD vs. patient: $27 \%$ vs. $19 \% ; p=0.13$ ) (Table 1). The value of total cholesterol was higher in the patient group (CAD vs. patient: $155.49 \pm 36.23$ vs. $166.02 \pm 32.66 ; p=0.01$ ) (Table 1 ), which might be related to the greater high-density lipoprotein cholesterol level in these patients (CAD vs. patient: $38.4 \pm 12.31$ vs. $43.58 \pm 10.92 ; p=0.04$ ) (Table 1 ). Because the pharmacotherapy data were not available in the present study, it could not be verified whether the CAD group was characterized by lower DBP and cholesterol levels due to treatment.

The exosomes were extracted from both groups using the precipitation method for comparison. Interestingly, the amounts of exosomes were greater in the patient group (size $\leq 100 \mathrm{~nm}$, CAD vs. patient: $62,924.22 \pm 54,572.57$ vs. $76,339.77 \pm 48,357 ; p<0.001$ ) (Table 2). Other sizes of extracellular vesicles more than $100 \mathrm{~nm}$ are presented as well to show the distribution of the extracellular vesicles in detail in Table 2. Since patients in the CAD group were older and both groups were predominantly male, we performed multiple linear regression analysis to account for these co-variables. We found that age was an independent factor that correlated negatively with the change in the amounts of exosomes 
(size $\leq 100 \mathrm{~nm}$; increasing by one year was associated with decreasing 507.29 million particles $/ \mathrm{mL}, p=0.03$ ) (Table 3 ). However, other factors such as sex, HTN, and DM were not significantly associated with the alteration of exosome amounts (all $p>0.05$ ) (Table 3).

Table 1. Clinical baseline characteristics of the total population.

\begin{tabular}{|c|c|c|c|}
\hline & CAD (n = 214) & Patient $(n=140)$ & \multirow{2}{*}{$p$-Value } \\
\hline & Mean \pm SD & Mean \pm SD & \\
\hline Age (y) & $66.83 \pm 10.96$ & $60.82 \pm 11.67$ & $<0.001$ \\
\hline Male, N (\%) & $165(77 \%)$ & $96(69 \%)$ & 0.08 \\
\hline Waist (cm) & $74.72 \pm 20.16$ & $82.67 \pm 12.29$ & $<0.001$ \\
\hline $\mathrm{BMI}\left(\mathrm{kg} / \mathrm{m}^{2}\right)$ & $26.04 \pm 3.93$ & $26.75 \pm 4.00$ & 0.1 \\
\hline $\mathrm{SBP}(\mathrm{mm} \mathrm{Hg})$ & $135.43 \pm 26.68$ & $138.98 \pm 24.64$ & 0.21 \\
\hline $\mathrm{DBP}(\mathrm{mm} \mathrm{Hg})$ & $71.68 \pm 14.56$ & $75.48 \pm 13.01$ & 0.01 \\
\hline HTN & $150(70 \%)$ & $75(54 \%)$ & 0.03 \\
\hline $\mathrm{DM}$ & $98(46 \%)$ & $37(26 \%)$ & 0.02 \\
\hline Proteinuria & $58(27 \%)$ & $27(19 \%)$ & 0.13 \\
\hline Smoking & $53(25 \%)$ & $43(31 \%)$ & 0.23 \\
\hline Alcohol & $36(17 \%)$ & $26(19 \%)$ & 0.67 \\
\hline $\mathrm{Hb}(\mathrm{g} / \mathrm{dL})$ & $13.47 \pm 2.04$ & $13.95 \pm 1.67$ & 0.02 \\
\hline Glu AC (mg/dL) & $139.51 \pm 56.01$ & $122.67 \pm 40.58$ & $<0.001$ \\
\hline $\mathrm{HbA} 1 \mathrm{C}(\mathrm{mg} / \mathrm{dL})$ & $6.47 \pm 1.38$ & $6.38 \pm 1.75$ & 0.84 \\
\hline GOT (U/L) & $20.77 \pm 6.48$ & $22.06 \pm 6.82$ & 0.31 \\
\hline GPT (U/L) & $27.61 \pm 6.17$ & $29.66 \pm 8.08$ & 0.22 \\
\hline BUN (mg/dL) & $23.06 \pm 14.94$ & $17.43 \pm 4.75$ & $<0.001$ \\
\hline $\mathrm{Cr}(\mathrm{mg} / \mathrm{dL})$ & $1.47 \pm 1.86$ & $0.99 \pm 0.57$ & $<0.001$ \\
\hline $\mathrm{Na}(\mathrm{mmol} / \mathrm{L})$ & $137.29 \pm 2.79$ & $137.6 \pm 2.34$ & 0.26 \\
\hline $\mathrm{K}(\mathrm{mmol} / \mathrm{L})$ & $4.05 \pm 0.44$ & $4.02 \pm 0.36$ & 0.52 \\
\hline $\mathrm{CHO}(\mathrm{mg} / \mathrm{dL})$ & $155.49 \pm 36.23$ & $166.02 \pm 32.66$ & 0.01 \\
\hline $\mathrm{TG}(\mathrm{mg} / \mathrm{dL})$ & $158.04 \pm 104.89$ & $157.8 \pm 83.05$ & 0.98 \\
\hline $\mathrm{LDL}(\mathrm{mg} / \mathrm{dL})$ & $91.48 \pm 29.24$ & $97.09 \pm 27.88$ & 0.08 \\
\hline HDL (mg/dL) & $38.4 \pm 12.31$ & $43.58 \pm 10.92$ & 0.04 \\
\hline $\mathrm{CRP}(\mathrm{mg} / \mathrm{dL})$ & $4.23 \pm 6.97$ & $0.13 \pm 0.05$ & $<0.001$ \\
\hline
\end{tabular}

All results are expressed as means and standard deviations (mean $\pm \mathrm{SD}$ ) for both men and women. CAD, coronary artery disease; $\mathrm{BH}$, body height; $\mathrm{BW}$, body weight; $\mathrm{SBP}$, systolic blood pressure; DBP, diastolic blood pressure; $\mathrm{HTN}$, hypertension; DM, diabetes mellitus; $\mathrm{Hb}$, hemoglobin; Glu AC, fasting blood glucose; $\mathrm{HbA1C}$, hemoglobin A1c; GOT, glutamic oxaloacetic transaminase; GPT, glutamic pyruvic transaminase; BUN, blood urea nitrogen; $\mathrm{Cr}$, creatinine; $\mathrm{Na}$, sodium; $\mathrm{K}$, potassium; $\mathrm{CHO}$, cholesterol; $\mathrm{TG}$, triglyceride; $\mathrm{LDL}$, low-density lipoprotein cholesterol; HDL, high-density lipoprotein cholesterol; CRP, C-reactive protein.

After the exosomes were extracted, 52 consecutive samples were further selected from each of the CAD and patient groups for next-generation sequencing (NGS). The basic characteristics of the 104 patients from which the NGS samples were obtained are presented in Supplementary Table S1. When comparing the two subgroups selected for NGS, the mean age of the CAD subgroup was still older than the patient subgroup (CAD vs. patient: $65.04 \pm 10.68$ vs. $60.65 \pm 11.26 ; p=0.04$ ) (Supplementary Table S1). Both subgroups were predominantly male and had similar profiles for physical appearance, blood pressure, systemic diseases (DM and HTN), and social behavior (Supplementary Table S1). The results of the biochemistry examinations of both subgroups were also within the normal limits (Supplementary Table S1). The amounts of exosomes obtained from both selected 
groups were also compared, and no significant differences were found after the co-variables were adjusted in the multiple linear regression analysis (Supplementary Tables S2 and S3).

Table 2. The amounts of exosomes from the total population.

\begin{tabular}{|c|c|c|c|c|c|c|c|}
\hline & \multicolumn{3}{|c|}{ CAD $(n=214)$} & \multicolumn{3}{|c|}{ Patient (n = 140) } & \multirow{2}{*}{$\begin{array}{c}\text { Mann-Whitney } \\
\text { U-Test }\end{array}$} \\
\hline & Mean \pm SD & Median & IQR & Mean \pm SD & Median & IQR & \\
\hline Size (nm) & \multicolumn{3}{|c|}{ Million Particles/mL } & \multicolumn{3}{|c|}{ Million Particles/mL } & $p$-Value \\
\hline$\leq 100$ & $62,924.22 \pm 54,572.57$ & $49,480.92$ & $68,653.44$ & $76,339.77 \pm 48,357$ & $67,434.48$ & $65,549.61$ & $<0.001$ \\
\hline $100 \sim 200$ & $9,631.07 \pm 10,120.11$ & 6674.68 & $12,533.43$ & $12,523.47 \pm 8767.05$ & $11,719.74$ & $13,367.65$ & $<0.001$ \\
\hline$\leq 200$ & $79,914.23 \pm 64,822.03$ & $71,042.42$ & $76,675.62$ & $94,487.55 \pm 55,614.36$ & $84,257.18$ & $71,795.00$ & $<0.001$ \\
\hline $200 \sim 500$ & $101.33 \pm 160.23$ & 34.65 & 112.62 & $124.04 \pm 148.8$ & 70.06 & 155.63 & 0.03 \\
\hline
\end{tabular}

CAD, coronary artery disease; $\mathrm{SD}$, standard deviation; IQR, interquartile range.

Table 3. Multiple linear regression for the amounts of exosomes from the total population.

\begin{tabular}{ccccc}
\hline & \multicolumn{2}{c}{ Size $\leq \mathbf{1 0 0}(\mathbf{n m})$} & \multicolumn{2}{c}{ Size $\leq \mathbf{2 0 0}(\mathbf{n m})$} \\
\cline { 2 - 5 } & $\boldsymbol{\beta}$ (Million Particles $/ \mathbf{m L})$ & $p$-value & $\beta$ (Million Particles $/ \mathbf{m L})$ & $p$-Value \\
\hline CAD & -4218.59 & 0.02 & -5023.20 & 0.03 \\
\hline Age & -507.29 & 0.03 & -635.71 & 0.03 \\
\hline Sex & 4645.49 & 0.46 & 837.41 & 0.91 \\
\hline HTN & 1967.54 & 0.58 & 324.58 & 0.94 \\
\hline DM & 3113.07 & 0.52 & 5368.82 & 0.37 \\
\hline
\end{tabular}

CAD, coronary artery disease; HTN, hypertension; DM, diabetes mellitus.

To investigate the possible relationship between stable three-vessel CAD and exosomes, the alteration in exosomal miRNA expression was observed by NGS using samples of the selected subgroups. After comparative exosomal miRNA analysis, eight miRNAs showing up- or down-regulations in stable three-vessel CAD were identified among the 454 exosomal miRNAs examined (Table 4). Among the miRNAs with altered expressions, miRNA-382-3p, miRNA-432-5p, miRNA-200a-3p, and miRNA-3613-3p were noted to be up-regulated in the selected CAD subgroup based on the Mann-Whitney U-test (Table 4). On the contrary, miRNA-125a-5p, miRNA-185-5p, miRNA-151a-3p, and miRNA-328-3p were found to be down-regulated in the selected CAD subgroup (Table 4).

Table 4. Comparison of exosomal miRNAs from the selected CAD and patient groups.

\begin{tabular}{cccccccc}
\hline \multirow{2}{*}{ miRNA Locus } & \multicolumn{3}{c}{ Selected CAD } & \multicolumn{3}{c}{ Selected Patient } \\
\cline { 2 - 8 } & \multicolumn{3}{c}{$\mathbf{N}=\mathbf{4 6}$} & \multicolumn{2}{c}{ M2 } & \multicolumn{2}{c}{ Mann-Whitney U-Test } \\
\cline { 2 - 8 } & Mean \pm SD & Median & IQR & Mean \pm SD & Median & IQR & $p$-Value \\
\hline miRNA-382-3p & $15.76 \pm 35.93$ & 0.00 & 0.00 & $3.5 \pm 17.17$ & 0.00 & 0.00 & 0.02 \\
\hline miRNA-432-5p & $11.05 \pm 29.4$ & 0.00 & 0.00 & 0 & 0.00 & 0.00 & 0.01 \\
\hline miRNA-200a-3p & $11.57 \pm 31.7$ & 0.00 & 0.00 & $0.85 \pm 5.48$ & 0.00 & 0.00 & 0.03 \\
\hline miRNA-3613-3p & $21.96 \pm 54.82$ & 0.00 & 0.00 & $1.25 \pm 8.09$ & 0.00 & 0.00 & 0.02 \\
\hline miRNA-125a-5p & $765.26 \pm 1661.5$ & 0.00 & 773.54 & $1945.61 \pm 2551.44$ & 242.74 & 3622.49 & 0.01 \\
\hline miRNA-185-5p & $26.83 \pm 50.77$ & 0.00 & 39.21 & $55.25 \pm 74.74$ & 0.00 & 92.30 & 0.05 \\
\hline miRNA-151a-3p & $502.46 \pm 522.85$ & 339.82 & 589.45 & $821.19 \pm 745.74$ & 510.97 & 1030.08 & 0.02 \\
\hline miRNA-328-3p & $2.63 \pm 12.8$ & 0.00 & 0.00 & $19.5 \pm 45.89$ & 0.00 & 0.00 & 0.05 \\
\hline
\end{tabular}

$\mathrm{CAD}$, coronary artery disease; miRNA, micro ribonucleic acid; SD, standard deviation; IQR, interquartile range.

\section{Discussion}

To summarize, there were several important findings and implication of this study: (1) This is by far the largest study utilizing exosomes directly extracted from coronary arteries to investigate the association between exosomes and stable three-vessel CAD. 
(2) This is also the first study that verifies both stable three-vessel CAD and age as the independent factors associated with changes in the amounts of exosomes. (3) The amounts of exosomes decreased in patients with three-vessel CAD as compared to patients without CAD. (4) The number of exosomes decreased with age, while sex, HTN, and DM did not affect the number of exosomes. (5) We identified altered expression in particular miRNAs that may be associated with the pathogenesis of stable three-vessel CAD. (6) The up-regulated miRNAs in stable three-vessel CAD were miRNA-382-3p, miRNA-432-5p, miRNA-200a-3p, and miRNA-3613-3p. (7) The down-regulated miRNAs in stable threevessel CAD were miRNA-125a-5p, miRNA-185-5p, miRNA-151a-3p, and miRNA-328-3p.

\subsection{Variation in the Amounts of Exosomes Associated with CAD and Age}

We extracted exosomes directly from the coronary arterial blood and compared the amounts of exosomes between patients with and without CAD using the precipitation method. Strikingly, there were much greater amounts of exosomes obtained from the patient group as compared to the stable three-vessel CAD group (all $p<0.05$ ). Even after the covariates were adjusted, stable three-vessel CAD was still negatively associated with the number of exosomes. It has been suggested in past studies that cardiomyocytes may increase the secretion of exosomes in response to stress, such as hypoxia, inflammation, or injury [14]. However, our results showed a decreased number of exosomes in patients with three-vessel CAD, which was different from prior presumptions. Therefore, it is reasonable to suspect that instead of the amount, it may actually be the contents of the exosomes that vary under pathological conditions such as ischemic stimuli. Additionally, we noted that the amounts of exosomes were negatively associated with the aging process, which is a novel finding that has not been reported before (Table 3).

\subsection{Exosomal miRNAs as the Novel Biomarkers for CAD}

miRNAs are endogenous, single-stranded, short, non-coding RNA molecules approximately $22 \mathrm{nt}$ in length. They finetune gene expression by binding to the $3^{\prime}$ untranslated region of the mRNA at a complementary region located approximately at bases 2-8 from the $5^{\prime}$ end, termed the seed region. By matching complementary sequences within the target mRNA molecule, miRNAs could control the expression of functional and biological pathways [15]. Mostly, miRNAs mediate post-transcriptional gene expression by inhibiting mRNA translation or promoting its degradation, resulting in the direct or indirect reduction of protein production. Many biological processes, such as cell proliferation, differentiation, and apoptosis, are reported to be regulated by miRNAs [16]. Thus, miRNAs are considered to play an important role in intercellular communication with relevant roles in various physiological and pathological processes [17].

In comparison with the plasma miRNAs, recent research has revealed that miRNAs within exosomes can remain in a more durable and stable state since the packaged exosomal miRNAs can escape from nuclease degradation under the protection of the lipid bilayer membrane of the exosomes [18]. Moreover, exosomal miRNAs are more sensitive at predicting cardiovascular diseases as compared to circulating plasma miRNAs. For instance, the sensitivity of serum miRNA-208a for ACS diagnosis was inferior to that of exosomal miRNA-208a. The expression of exosomal miRNA-146a was significantly up-regulated in patients with heart failure, and that association was not found from the circulating plasma miRNA-146a. Therefore, exosomal miRNAs have greater potential for investigating cardiovascular diseases than circulating plasma miRNAs [19]. To the best of our knowledge, the current study is the largest study to explore the varied expression of exosomal miRNAs in stable three-vessel CAD by NGS. Most importantly, we successfully demonstrated the upand down-regulation of particular exosomal miRNAs that may serve as future biomarkers for stable three-vessel CAD. 


\subsection{Association between Exosomal miRNAs and CAD}

Our study possesses several strengths as compared to prior studies. In the study conducted by Zhang et al., eight miRNAs known to be involved in the pathogenesis of cardiovascular diseases (miR-192-5p, miR-148b-3p, miR-125a-3p, miR-942-5p, miR-149$5 p$, miR-32-5p, miR-144- 3p, and miR-142-5p) were selected to investigate the association between exosomal miRNAs and CAD [19]. Since only the eight candidate miRNAs were examined, other previously unidentified exosomal miRNAs associated with CAD were likely missed in their study. In addition, instead of using peripheral blood samples, blood samples collected directly from the coronary arteries were used in this study for exosome extraction. Furthermore, a much greater number of patients were recruited in our work, indicating a better power to detect the relationship between exosomal miRNAs and CAD.

Previously, age and sex were covariates that posed challenges for analysis due to small sample sizes $[19,20]$. Su et al. used exosomal miRNAs to evaluate the pathophysiological progression from CAD to acute myocardial infraction (AMI) [18]. However, only six AMI and six matching CAD patients were enrolled for miRNA screening, making the age and sex adjustment unavailable in their study [18]. Of note, possible confounders such as age, sex, HTN, and DM were adjusted in the present study to evaluate the association of miRNAs and stable three-vessel CAD, which had not been performed before. However, there might be differences regarding miRNAs between stable CAD, old myocardial infarction, and ACS. Further studies about this issue are needed.

\subsection{Association among Exosomal miRNAs, Exosome-Originated Protein, and CAD}

Notably, Moreira-Costa et al. performed a literature search and network analysis to evaluate the interaction between exosomes and cardiovascular diseases [21]. For CAD patients, up-regulated exosomal proteins, including fibrinogen beta/gamma chain, interalpha-trypsin inhibitor heavy chain, and alpha-1 antichymotrypsin, were assessed as putative protein biomarkers. Several down-regulated proteins were also considered relevant to CAD, such as albumin, clusterin, and vitamin-D-binding protein [21]. While our findings provide essential information for the involvement of exosomal miRNAs in stable three-vessel CAD, their results shed light on the possible validation of miRNAs and exosome-originated protein in combination with the novel biomarkers of CAD [21]. Future investigation of exosomal miRNAs in association with relevant proteins may help further elucidate the signaling pathways involved in CAD.

\subsection{The Up-Regulated Exosomal miRNAs in CAD}

Four miRNAs were found with increased expression in there-vessel CAD in this study. Among them, miRNA-382-3p has previously been noted to be associated with the reduction of L-type $\mathrm{Ca}^{2+}$ channel density and pro-arrhythmogenic effects [22]. However, the association between miRNA-382-3p and CAD has not been described. As for miRNA432-5p, Gao et al. found an increased level of miRNA-432-5p in the peripheral blood mononuclear cells of CAD patients, which was consistent with our findings [23]. The other two miRNAs, miRNA-200a-3p and miRNA-3613-3p, were first identified to be related to the signaling pathways of CAD in our study. Future studies focusing on these miRNAs are needed to verify their association with CAD.

\subsection{The Down-Regulated Exosomal miRNAs in CAD}

We also found four miRNAs that were down-regulated in CAD. miRNA-125a-5p is highly expressed in vascular endothelial cells and plays a role in modulating the inflammatory response within macrophages by repressing a negative regulator of NF-kB signaling pathways [24]. Moreover, it has been reported to modulate lipid uptake and decrease the secretion of inflammatory cytokines in oxidized low-density lipoprotein (oxLDL)-stimulated monocyte-derived macrophages [25]. Therefore, by suppressing oxLDL-induced endothelin1 (ET-1) expression, miRNA-125a-5p plays a protective role against the development of atherosclerosis [26]. Our findings were consistent with the study by Hao et al., in which 
miRNA-125a-5p, miRNA-155, and miRNA-199a/b-3p were observed to be down-regulated with the increased expression of ET-1 and played a crucial role in coronary atherosclerosis [26]. For miRNA-185-5p, it was proven to be involved in the cell proliferation, metastasis, and inflammation of various tumors, but its regulatory role in the progression of CAD remains elusive [25]. As for miRNA-151a-3p and miRNA-328-3p, they were also first reported to be down-regulated in CAD in the current study, and the underlying molecular mechanisms remain to be explored.

In conclusion, this is the first study utilizing exosomes extracted from coronary arterial blood to demonstrate the decreased amounts of exosomes in stable three-vessel CAD patients and aged populations. We also identified eight exosomal miRNAs with altered expression in stable three-vessel CAD. These findings provide novel insights into the molecular pathophysiology of CAD and may serve as the basis for exploring potential biomarkers and predictors of CAD.

\section{Limitations}

In the present study, whether the miRNAs highlighted could be used as biomarkers or are merely associated by chance remains to be conclusively proven. Previously, increased levels of miRNA-432-5p were associated with CAD occurrence, and miRNA-125a-5p had been found to play a protective role against the development of atherosclerosis [23-26]. Our findings could be regarded as their validation. There were several novel miRNAs that had not been found before in relation to stable CAD in this study. We are preparing another cohort study specifically focused on stable CAD to validate these miRNAs. Further cellular studies about the over-expression or knock-out of these miRNAs can also clarify the signaling pathways of these miRNAs in association with the pathogenesis of stable CAD. Therefore, our findings should be interpreted carefully to avoid false-positive signals. Moreover, there might be differences regarding miRNAs between stable CAD, old MI, and ACS. Further large sample size cohort studies about this issue might clarify this. Finally, this was a cross-sectional cohort study, and we aimed to find the miRNAs connected with severe CAD. Even though we also enrolled patients with stable one-vessel or two-vessel CAD, we did not compare this with patient participants. The miRNAs were analyzed immediately after three-vessel CAD was confirmed by coronary angiography. Therefore, we could not conclude that these miRNAs are related to the pathophysiological progress from one-vessel to three-vessel CAD.

\section{Conclusions}

In summary, this was the first study to use exosomes extracted from blood samples taken from coronary arteries to show that exosomes' amounts decreased in stable threevessel CAD patients and during the aging process. We also identified eight exosomal miRNA profiles to serve as potential biomarkers and predictors of stable CAD. These findings provide novel insights into the molecular pathophysiology of CAD.

Supplementary Materials: The following supporting information can be downloaded at: https: / / www.mdpi.com/article/10.3390/cells11010098/s1, Table S1: Clinical baseline characteristics of the selected population for NGS; Table S2: The amounts of exosomes from the selected population for NGS; Table S3: Multiple linear regression for the amounts of exosomes from the selected population for NGS; Figure S1: Study workflow.

Author Contributions: Regarding the contribution of each author, S.-N.C. analyzed the data and worked on the drafting of the manuscript. J.-J.C., J.-H.W., Y.-T.C., J.-W.C., C.-H.C., C.-J.L. and M.-T.L. analyzed the data. Y.-C.C., C.L., J.-J.H. and W.-P.L. interpreted the data. J.-W.L. designed the whole study and supervised every step of the work. All authors revised the manuscript critically for important and intellectual contents, approved the final version to be published, and agreed to be accountable for the accuracy and integrity of their parts of the work. All authors have read and agreed to the published version of the manuscript. 
Funding: This work was supported by a grant from the National Taiwan University Hospital Yun-Lin Branch (NTUHYL107.X005 and 110.X004). None of the authors disclose any financial or personal relationships with other people or organizations that could inappropriately influence (bias) their work.

Institutional Review Board Statement: This study was approved by the Ethics Committee and Institutional Review Board on Human Research of the Medical Research Department of National Taiwan University Hospital, Taipei, Taiwan (201811060RINC/202001100RINB: 2018/11/2020/01), and adhered to the provisions set out in the Declaration of Helsinki.

Informed Consent Statement: All subjects provided written informed consent before participating in the study.

Acknowledgments: We immensely thank the facility staff.

Conflicts of Interest: The authors declare that they have no conflict of interest.

\section{References}

1. Chen, G.H.; Xu, J.; Yang, Y.J. Exosomes: Promising sacks for treating ischemic heart disease? Am. J. Physiol. Heart Circ. Physiol. 2017, 313, H508-H523. [CrossRef]

2. Terrasini, N.; Lionetti, V. Exosomes in critical illness. Crit. Care Med. 2017, 45, 1054-1060. [CrossRef] [PubMed]

3. Perrotta, I.; Aquila, S. Exosomes in human atherosclerosis: An ultrastructural analysis study. Ultrastruct. Pathol. 2016, 40, 101-106. [CrossRef] [PubMed]

4. Barderas, M.G.; Vivanco, F.; Alvarez-Llamas, G. Vascular proteomics. Methods Mol. Biol. 2013, 1000, 1-20. [CrossRef] [PubMed]

5. Chistiakov, D.A.; Orekhov, A.N.; Bobryshev, Y.V. Cardiac extracellular vesicles in normal and infarcted heart. Int. J. Mol. Sci. 2016, 17, 63. [CrossRef] [PubMed]

6. $\quad$ Glinge, C.; Clauss, S.; Boddum, K.; Jabbari, R.; Jabbari, J.; Risgaard, B.; Tomsits, P.; Hildebrand, B.; Kääb, S.; Wakili, R.; et al. Stability of circulating blood-based microRNAs-Pre-analytic methodological considerations. PLoS ONE 2017, 12, e0167969. [CrossRef] [PubMed]

7. Arnold, S.V.; Bhatt, D.L.; Barsness, G.W.; Beatty, A.L.; Deedwania, P.C.; Inzucchi, S.E.; Kosiborod, M.; Leiter, L.A.; Lipska, K.J.; Newman, J.D.; et al. Clinical management of stable coronary artery disease in patients with type 2 diabetes mellitus: A scientific statement from the american heart association. Circulation 2020, 141, e779-e806. [CrossRef] [PubMed]

8. Máchal, J.; Pávková-Goldbergová, M.; Hlinomaz, O.; Groch, L.; Vašků, A. Patients with chronic three-vessel disease in a 15-year follow-up study: Genetic and non-genetic predictors of survival. Medicine 2014, 93, e278. [CrossRef] [PubMed]

9. Biosciences. ExoQuick ${ }^{\circledR}$ Exosome Isolation and RNA Purification Kits User Manual, 2nd ed.; Oxford University Press: Oxford, UK, 2018.

10. Ekström, K.; Omar, O.; Granéli, C.; Wang, X.; Vazirisani, F.; Thomsen, P. Monocyte exosomes stimulate the osteogenic gene expression of mesenchymal stem cells. PLOS ONE 2013, 8, e75227. [CrossRef]

11. Nicole Weit, A.B.; Kapinsky, M. High Content DuraClone Reagents on the CytoFLEX Flow Cytometer: Instrument Setup Using the CytoFLEX Compensation Library; Beckman Coulter GmbH: Vienna, Austria, 2015.

12. Yoshioka, Y.; Konishi, Y.; Kosaka, N.; Katsuda, T.; Kato, T.; Ochiya, T. Comparative marker analysis of extracellular vesicles in different human cancer types. J. Extracell Vesicles 2013, 2. [CrossRef] [PubMed]

13. Moll, P.; Ante, M.; Seitz, A.; Reda, T. QuantSeq 3' mRNA sequencing for RNA quantification. Nat. Methods 2014, 11, 1-3. [CrossRef]

14. Tokarz, A.; Szuscik, I.; Kusnierz-Cabala, B.; Kapusta, M.; Konkolewska, M.; Zurakowski, A.; Georgescu, A.; Stepien, E. Extracellular vesicles participate in the transport of cytokines and angiogenic factors in diabetic patients with ocular complications. Folia Med. Cracov 2015, 55, 35-48.

15. Dudink, E.; Florijn, B.; Weijs, B.; Duijs, J.; Luermans, J.; Peeters, F.; Schurgers, L.; Wildberger, J.; Schotten, U.; Bijkerk, R.; et al. Vascular calcification and not arrhythmia in idiopathic atrial fibrillation associates with sex differences in diabetic microvascular injury miRNA profiles. Microrna 2019, 8, 127-134. [CrossRef] [PubMed]

16. Liu, Q.; Li, H.; Wang, N.; Chen, H.; Wang, J. Expression of miR-126 and miR-508-5p in endothelial progenitor cells is associated with the prognosis of chronic heart failure patients. Int. J. Cardiol. 2013, 168, 2082-2088. [CrossRef]

17. Lozano-Prieto, M.; Adlam, D.; García-Guimaraes, M.; Sanz-García, A.; Vera-Tomé, P.; Rivero, F.; Cuesta, J.; Bastante, T.; Baranowska-Clarke, A.A.; Vara, A.; et al. Differential miRNAs in acute spontaneous coronary artery dissection: Pathophysiological insights from a potential biomarker. EBioMedicine 2021, 66, 103338. [CrossRef]

18. Su, J.; Li, J.; Yu, Q.; Wang, J.; Li, X.; Yang, J.; Xu, J.; Liu, Y.; Xu, Z.; Ji, L.; et al. Exosomal miRNAs as potential biomarkers for acute myocardial infarction. IUBMB Life 2020, 72, 384-400. [CrossRef]

19. Zhang, P.; Liang, T.; Chen, Y.; Wang, X.; Wu, T.; Xie, Z.; Luo, J.; Yu, Y.; Yu, H. Circulating exosomal miRNAs as novel biomarkers for stable coronary artery disease. Biomed. Res. Int. 2020, 2020, 3593962. [CrossRef]

20. He, G.D.; Huang, Y.Q.; Liu, L.; Huang, J.Y.; Lo, K.; Yu, Y.L.; Chen, C.L.; Zhang, B.; Feng, Y.Q. Association of circulating, inflammatory-response exosomal mRNAs with acute myocardial infarction. Front. Cardiovasc. Med. 2021, 8, 712061. [CrossRef] 
21. Moreira-Costa, L.; Barros, A.S.; Lourenço, A.P.; Leite-Moreira, A.F.; Nogueira-Ferreira, R.; Thongboonkerd, V.; Vitorino, R. Exosome-derived mediators as potential biomarkers for cardiovascular diseases: A network approach. Proteomes $2021,9,8$. [CrossRef] [PubMed]

22. Dlouha, D.; Prochazkova, I.; Eretova, Z.; Hubacek, J.A.; Parikova, A.; Pitha, J. Influence of lipoprotein apheresis on circulating plasma levels of miRNAs in patients with high Lp(a). Atheroscler. Suppl. 2019, 40, 12-16. [CrossRef]

23. Gao, J.; Liu, J.; Zhang, Y.; Guan, B.; Qu, H.; Chai, H.; Wang, W.; Ma, X.; Shi, D. PBMCs-Derived microRNA signature as a prethrombotic status discriminator in stable coronary artery disease. Thromb. Haemost. 2020, 120, 121-131. [CrossRef]

24. Ormseth, M.J.; Solus, J.F.; Vickers, K.C.; Oeser, A.M.; Raggi, P.; Stein, C.M. Utility of select plasma microrna for disease and cardiovascular risk assessment in patients with rheumatoid arthritis. J. Rheumatol. 2015, 42, 1746-1751. [CrossRef] [PubMed]

25. Li, M.; Qi, L.; Li, Y.; Zhang, S.; Lin, L.; Zhou, L.; Han, W.; Qu, X.; Cai, J.; Ye, M.; et al. Association of pericardiac adipose tissue with coronary artery disease. Front. Endocrinol. 2021, 12, 724859. [CrossRef] [PubMed]

26. Hao, L.; Wang, X.G.; Cheng, J.D.; You, S.Z.; Ma, S.H.; Zhong, X.; Quan, L.; Luo, B. The up-regulation of endothelin-1 and down-regulation of miRNA-125a-5p, -155, and -199a/b-3p in human atherosclerotic coronary artery. Cardiovasc. Pathol. 2014, 23, 217-223. [CrossRef] [PubMed] 